hep-th/0208187

IPM/P-2002/034

\title{
Branes in Time-Dependent Backgrounds and AdS/CFT Correspondence
}

\author{
Mohsen Alishahiha1 and Shahrokh Parvizil \\ Institute for Studies in Theoretical Physics and Mathematics (IPM) \\ P.O. Box 19395-5531, Tehran, Iran
}

\begin{abstract}
We study supergravity solutions of Dp-branes in the time-dependent orbifold background. We show that worldvolume theories decouple from the bulk gravity for $p$ less than six. Along AdS/CFT correspondence, these solutions could provide the gravity description of noncommutative field theory with time-dependent noncommutative parameter. Type II NS5-brane (M5-brane) in the presence of $\mathrm{RR} n$-form for $n=0, \cdots, 4$ (C field) in this time-dependent background have also been studied.
\end{abstract}

\footnotetext{
${ }^{1}$ e-mail: alishah@ipm.ir

${ }^{2}$ e-mail: parvizi@theory.ipm.ac.ir
} 


\section{Introduction}

Recently string theory in time-dependent backgrounds has attracted much interest (recent papers on this subject include [1]-29]). This is because in order to apply the string theory in cosmology we would need to know the string theory in time-dependent backgrounds. As an example one can consider orbifold quotients of Minkowski space-time which non-trivially act on both space and time 11, 3, 4, 5, 7, 8, 14, 16, 22, 23, 28, 29. We note, however, that in general orbifolds of space-time leads to singularities corresponding to the closed light-like loops. Nevertheless one can resolve these singularities by making use of the null-brane backgrounds [3, 22, 23, 29] which is obtained form the space-time orbifold together with an extra shift. In the next section we review this geometry.

This null-brane background preserves one-half of the original flat space supersymmetry. It has also a null Killing vector which makes it possible to use light-cone quantization. The stability of this geometry has also been studied in [21, 23, 24].

Open string sector in this background has recently been considered [28, 29]. This, for example, can be done by using D-branes for probing the geometry. D3-brane probing this background has been studied in [28] where the authors argued that the D3-brane worldvolume decouples from the bulk gravity leading to a noncommutative gauge theory with time-dependent noncommutativity parameter. This is the aim of this article to generalize this consideration for other D-branes as well as NS5brane. In the NS5-brane case the situation reminisces the ODp-theory, namely we will find NS5-brane solution in the presence of RR $n$-form for $n=0, \cdots, 4$ in the time-dependent background. We shall also consider M5-brane in an 11-dimensional time-dependent background which can be obtained in the same way as the one in string theory. Namely we could start from an 11-dimensional null-brane geometry of M-theory. The theory on the corresponding M5-brane can be thought as a new deformation of $(0,2)$ theory. This system is very similar to OM-theory [30].

The organization of the paper is as follows. In section 2 we will review the null-brane background and then we shall probe this background by Dp-brane for $2<p \leq 6$. We will argue that the worldvolume theory decouples from gravity for $p \leq 5$ and therefore this background would provide a gravity description for noncommutative field theory with time-dependent noncommutativity parameter. In section 3 we will consider the near horizon limit of the background obtained in section 2. We shall briefly study the phase structure of the theory as well. In section 4 type II NS5-branes in the null-brane geometry are considered which lead to new deformation of the little string theory. In section 5 M5-brane probing 11dimensional null-brane geometry is studied. Section 6 is devoted to the comments and conclusions. 


\section{Supergravity solution}

In this section we shall briefly review the structure of null-brane geometry. Then in order to study the properties of this background in string theory we will probe the geometry by D-branes. This background could change the worldvolume theory of the brane leading, probably, to a new field theory.

We start with a 10-dimensional flat spacetime $R^{1,9}$ with metric

$$
d s^{2}=-2 d x^{+} d x^{-}+d x^{2}+d z^{2}+d \vec{Y}_{6}^{2},
$$

where $d \vec{Y}_{6}^{2}$ is the flat 6 -dimensional space. Now consider the orbifold obtained by identifying [14, 22]

$$
\left(\begin{array}{c}
x^{+} \\
x \\
x^{-} \\
z
\end{array}\right) \sim\left(\begin{array}{c}
x^{+} \\
x+2 \pi n x^{+} \\
x^{-}+2 \pi n x+2(\pi n)^{2} x^{+} \\
z+2 \pi n \beta
\end{array}\right), \quad n \in Z,
$$

while leaves $d \vec{Y}_{6}^{2}$ unchanged and $\beta$ is a constant. The above orbifold background is called the null-brane [3].

It is useful to introduce a new set of coordinates as following

$$
x^{+}=y^{+}, \quad x=y^{+} y, \quad x^{-}=y^{-}+\frac{1}{2} y^{+} y^{2},
$$

and other coordinates remain the same as before. In this coordinates the orbifold metric reads

$$
d s^{2}=-2 d y^{+} d y^{-}+\left(y^{+}\right)^{2} d y^{2}+d z^{2}+d \vec{Y}_{6}^{2},
$$

and the orbifold identification becomes

$$
\left(\begin{array}{c}
y^{+} \\
y \\
y^{-} \\
z
\end{array}\right) \sim\left(\begin{array}{c}
y^{+} \\
y+2 \pi n \\
y^{-} \\
z+2 \pi n \beta
\end{array}\right)
$$

Now we want to probe this background with a system of $N$ Dp-branes for $p>2$ with worldvolume directions $\left(y^{+}, y^{-}, y, z, x_{1} \cdots x_{p-3}\right)$ where $x_{i}$ 's are $(p-3)$ -

dimensional subspace of $\vec{Y}_{6}$. The procedure is very similar to that in [31] (see also [32]) to construct the supergravity dual of the noncommutative dipole theory. To do this we start with the flat Dp-brane in the $y$ coordinates

$$
\begin{aligned}
& d s^{2}=f^{-1 / 2}\left(-2 d y^{+} d y^{-}+\left(y^{+}\right)^{2} d y^{2}+d z^{2}+\sum_{i=1}^{p-3} d x_{i}^{2}\right)+f^{1 / 2}\left(d r^{2}+r^{2} d \Omega_{8-p}^{2}\right) \\
& e^{2 \phi}=f^{(3-p) / 2}, \quad d C_{+-y z 1 \cdots(p-3)} \sim \partial_{r} f^{-1}, \quad f=1+\frac{R^{7-p}}{r^{7-p}}
\end{aligned}
$$


where $R^{7-p}=c_{p} g_{s} N l_{s}^{7-p}$ with $c_{p}=2^{5-p} \pi^{\frac{5-p}{2}} \Gamma\left(\frac{7-p}{2}\right)$. Here $z$ is compactified on a circle of radius $\beta$. T-dualizing along $z$ coordinate leads to the $\mathrm{D}(\mathrm{p}-1)$-brane smeared over one direction

$$
\begin{aligned}
d s^{2} & =f^{-1 / 2}\left(-2 d y^{+} d y^{-}+\left(y^{+}\right)^{2} d y^{2}+\sum_{i=1}^{p-3} d x_{i}^{2}\right)+f^{1 / 2}\left(d z^{2}+d r^{2}+r^{2} d \Omega_{8-p}^{2}\right), \\
e^{2 \phi} & =f^{(4-p) / 2}, \quad d C_{+-y 1 \cdots(p-3)} \sim \partial_{r} f^{-1}, \quad f=1+\frac{R^{7-p}}{r^{7-p}} .
\end{aligned}
$$

We now twist $y$ coordinate so that under identification of $z$ one gets

$$
z \sim z+2 \pi \beta, \quad y \sim y+2 \pi
$$

One can define a new coordinate in which $y \rightarrow y-\frac{z}{\beta}$ such that the identification becomes trivial

$$
z \sim z+2 \pi \beta, \quad y \sim y .
$$

In this new coordinates the T-dualized metric (7) reads

$$
\begin{aligned}
d s^{2} & =f^{-1 / 2}\left(-2 d y^{+} d y^{-}+\sum_{i=1}^{p-3} d x_{i}^{2}\right)+f^{1 / 2}\left(d r^{2}+r^{2} d \Omega_{8-p}^{2}\right) \\
& +f^{-1 / 2}\left(y^{+}\right)^{2} d y^{2}+f^{1 / 2}\left(1+\frac{\left(y^{+}\right)^{2}}{\beta^{2} f}\right) d z^{2}+2 f^{-1 / 2} \frac{\left(y^{+}\right)^{2}}{\beta} d y d z
\end{aligned}
$$

T-dualizing back along $z$ direction and using the T-duality rules 33 we will find a supergravity solution of Dp-brane probing the orbifold background (4) as following

$$
\begin{aligned}
d s^{2} & =f^{-1 / 2}\left(-2 d y^{+} d y^{-}+h\left(\left(y^{+}\right)^{2} d y^{2}+d z^{2}\right)+\sum_{i=1}^{p-3} d x_{i}^{2}\right)+f^{1 / 2}\left(d r^{2}+r^{2} d \Omega_{8-p}^{2}\right) \\
e^{2 \phi} & =h f^{(3-p) / 2}, \quad h^{-1}=1+\frac{\left(y^{+}\right)^{2}}{\beta^{2} f}, \quad f=1+\frac{R^{7-p}}{r^{7-p}} \\
B_{y z} & =\frac{\left(y^{+}\right)^{2}}{\beta} h f^{-1}, \quad d C_{+-y z 1 \cdots(p-3)} \sim \partial_{r} f^{-1}
\end{aligned}
$$

In general given a supergravity solution of a system of branes, it is not clear whether the solution would give a well-defined description of some field theory. In fact, we must check and see whether there is a well-defined field theory on the brane worldvolume which decouples from the bulk gravity. To see this, one might calculate scattering amplitude for gravitons [36, 37] which can be done by computing the gravitons absorption cross section. If there is a limit (decoupling limit) where the

\footnotetext{
${ }^{3}$ The supersymmetry of branes in the null-brane geometry in string theory and M-theory was also studied in [34, 35. In fact the solution we have found here is dual to one of the possible reductions of the delocalised M2-brane discussed in section 3.2 of paper [34]. This solution is the special case of the reduction labelled $(\mathrm{A})$ in that section where the $\theta_{i}=0$. We would like to thank J. Figueroa-O'Farrill for bringing our attention to this point.
} 
gravitons absorption cross section vanishes, we have a field theory which decouples from the gravity. Using the same method as in 38] one can see that the background (11) give a well-defined theory in a decoupling limit for $p<6$.

More precisely, let us perturb the metric of the background (11) by

$$
g_{\mu \nu}=\bar{g}_{\mu \nu}+h_{\mu \nu} \quad \mu, \nu=0, \cdots, 9
$$

where by $\bar{g}_{\mu \nu}$ we denote the background metric (11) and $h_{\mu \nu}$ is the perturbation. We consider s-wave gravitons as following

$$
h_{\mu \nu}=\epsilon_{\mu \nu} \Psi\left(r, y^{+}, y^{-}\right), \quad \mu=0, \cdots, p .
$$

Using the linearized equations of motion of type II supergravity we find following equation for transverse gravitons

$$
\partial_{\mu}\left(\sqrt{-g} e^{-2 \phi} g^{\mu \nu} \partial_{\nu} \Psi\right)=0
$$

which leads to

$$
\partial_{r}\left(r^{(8-p)} \partial_{r} \Psi\right)-2 f r^{8-p} \partial_{+} \partial_{-} \Psi-\frac{r^{8-p}}{y^{+}} f \partial_{-} \Psi=0 .
$$

As an ansatz we take $\Psi\left(r, y^{+}, y^{-}\right)=h(r) Y\left(y^{+}, y^{-}\right)$. Thus we find two differential equations

$$
\partial_{r}^{2} h+\frac{8-p}{r} \partial_{r} h+\omega^{2} f h=0, \quad 2 \partial_{+} \partial_{-} Y+\frac{1}{y^{+}} \partial_{-} Y-\omega^{2} Y=0,
$$

The differential equation for $Y$ can be easily solved

$$
Y=\frac{1}{\sqrt{y^{+}}} e^{\frac{i \omega}{\sqrt{2}}\left(y^{+}+y^{-}\right)} .
$$

This time-dependence form of the solution reflects the fact that at large $r$ the space is not flat. From the radial equation one can read the potential by writing it in the form of a Schrödinger-like equation. Doing so we get the following Schrödinger-like equation

$$
\partial_{\rho}^{2} \varphi(\rho)+V_{p}(\rho) \varphi(\rho)=0
$$

where

$$
V_{p}(\rho)=-\left(1+\frac{c_{p} N g_{s}\left(\omega l_{s}\right)^{7-p}}{\rho^{7-p}}\right)+\frac{(8-p)(6-p)}{4 \rho^{2}}
$$

with $\rho=\omega r$.

This potential is the same as the one found in [38] for the ordinary D-branes as well as branes in the presence of B field. Therefore we conclude that we have a decoupled theory living on the worldvolume of Dp-brane for $p \leq 5$. 


\section{Decoupling limit}

In this section we study the near horizon limit of the supergravity solution (11). Having a decoupling limit, this could provide a gravity description of the theory along the AdS/CFT correspondence [39].

The decoupling limit of the background (11) is defined as a limit in which $l_{s} \rightarrow 0$ while keeping the following quantities fixed [28]

$$
u=\frac{r}{l_{s}^{2}}, \quad b=\frac{\beta}{l_{s}^{2}}, \quad \tilde{g}=g_{s} l_{s}^{p-3} .
$$

In this limit the supergravity solution (11) reads

$$
\begin{aligned}
l_{s}^{-2} d s^{2} & =\frac{u^{(7-p) / 2}}{\sqrt{g_{Y M}^{2} N}}\left(-2 d y^{+} d y^{-}+h\left(\left(y^{+}\right)^{2} d y^{2}+d z^{2}\right)+\sum_{i=1}^{p-3} d x_{i}^{2}\right) \\
& +\frac{\sqrt{g_{Y M}^{2} N}}{u^{(7-p) / 2}}\left(d u^{2}+u^{2} d \Omega_{8-p}^{2}\right), \\
e^{2 \phi} & =\tilde{g}^{2}\left(\frac{g_{Y M}^{2} N}{u^{7-p}}\right)^{\frac{3-p}{2}} h, \quad B_{y z}=\frac{l_{s}^{2}}{b} \frac{\left(y^{+}\right)^{2} u^{7-p}}{g_{Y M}^{2} N} h,
\end{aligned}
$$

where $h^{-1}=1+\frac{\left(y^{+}\right)^{2} u^{7-p}}{g_{Y M}^{2} b^{2} N}$ and $g_{Y M}^{2}=c_{p} \tilde{g}$.

Following 28] string theory on these backgrounds provides the gravity description of noncommutative gauge theories with nonconstant noncommutativity parameter in various dimensions.

The effective dimensionless coupling constant in the corresponding noncommutative field theory can be defined as following 40]

$$
g_{\mathrm{eff}}^{2} \sim g_{\mathrm{YM}}^{2} N u^{p-3}
$$

The scalar curvature of the metric in eq. (21) has the behavior

$$
l_{s}^{2} \mathcal{R} \sim \frac{1}{g_{\mathrm{eff}}} .
$$

Thus the perturbative calculation in noncommutative field theory can be trusted when $g_{\text {eff }} \ll 1$, while when $g_{\text {eff }} \gg 1$ the supergravity description is valid. We note also that the expression for dilaton in (21) can be recast to

$$
e^{\phi}=\frac{1}{N} g_{\mathrm{eff}}^{(7-p) / 4} h^{1 / 2}
$$

Keeping $g_{\text {eff }}$ and $h$ fixed we see from (24) that $e^{\phi} \sim 1 / N$. Therefore the string loop expansion corresponds to $1 / N$ expansion of noncommutative gauge theory.

Since the scalar curvature is time-independent, as far as the effective gauge coupling is concerned, the situation is the same as ordinary brane solution. But since 
the dilaton is time-dependent this will change the phase structure of the theory. In particular at given fixed energy the effective string coupling will change with time. There is a critical time $y_{c}^{+}=g_{Y M} b \sqrt{N} / u^{(7-p) / 2}$ where for $y^{+}>y_{c}^{+}$the noncommutative effects become important and the effective string coupling becomes

$$
e^{2 \phi} \sim \frac{b\left(g_{Y M}^{2} N\right)^{(9-p) / 2}}{N} \frac{u^{(p-5)(7-p) / 2}}{\left(y^{+}\right)^{2}} .
$$

Therefore the effective string coupling decreases in time, and thus under time evolution the gravity description becomes more applicable.

On the other hand at any given time the phase structure of the theory is the same as that in noncommutative field theory with constant noncommutativity parameter, namely those studied in [41] (see also [42]). The only difference is that the distinguished points where the description of the theory has to be changed is now time-dependent.

In the notation of [41] the dimensionless effective noncommutative parameter is given by

$$
a^{\mathrm{eff}}=\left(\frac{y^{+} u^{2}}{b g_{\mathrm{eff}}}\right)^{\frac{2}{7-p}} .
$$

At large distances $L \gg \sqrt{y^{+} / b g_{\text {eff }}}$ the noncommutative effects are small and the effective description of the worldvolume theory is in terms of a commutative field theory. Note that this distance is time-dependent which means that under time evolution of the theory the distance where the noncommutative effects are negligible becomes very large.

Form the expression of the dimensionless effective noncommutative parameter (26) one can read the noncommutative parameter seen by the gauge theory. In fact we get

$$
[y, z] \sim \frac{y^{+}}{b}
$$

which is in agreement with the field theory consideration [28].

\section{NS5-brane in the presence of RR field}

The worldvolume theory of NS5-branes in the presence of electric RR field decouples from bulk gravity leading to an interacting theory with light open Dp-brane. These theories are known as open Dp-brane theory or in short ODp-theory [43, 44]. The supergravity description of these theories have been studied in [45, 46, 47, 48].

In this section we shall study time-dependent background of type II NS5-brane in the presence of RR field. To do this, we start from D5-brane solution presented in the previous section. By making use of S-duality one can find the type IIB NS 
5-brane in the presence of RR 2-form $⿴$. Then a series of T-dualities will generate other possible RR fields. In fact by this procedure one gets

$$
\begin{aligned}
d s^{2} & =h^{-1 / 2}\left[-2 d y^{+} d y^{-}+h\left(\left(y^{+}\right)^{2} d y^{2}+\sum_{i=1}^{n} d x_{i}^{2}\right)+\sum_{j=n+1}^{3} d x_{j}^{2}+f\left(d r^{2}+r^{2} d \Omega_{3}^{2}\right)\right] \\
e^{2 \phi} & =f h^{(n-3) / 2}, \quad C_{y 1 \cdots n}^{(n+1)}=\frac{1}{g_{s}} \frac{\left(y^{+}\right)^{2}}{\beta} h f^{-1}, \quad f=1+\frac{N l_{s}^{2}}{r^{2}}
\end{aligned}
$$

which is type II NS5-brane in the presence of $\mathrm{RR}(n+1)$-form for $n=0,1,2,3$.

We note, however, that in the D5-brane solution (11) the metric is not symmetric under exchanging of $y \leftrightarrow z$.5 Therefore we have another choice of doing the series of T-dualities in which we have also make a T-duality along $y$ direction. Doing so we get another solution of type II NS5-brane in the presence of different possible RR fields as following

$$
\begin{aligned}
d s^{2} & =h^{-1 / 2}\left[-2 d y^{+} d y^{-}+\frac{d y^{2}}{\left(y^{+}\right)^{2}}+h \sum_{i=1}^{n} d x_{i}^{2}+\sum_{j=n+1}^{3} d x_{j}^{2}+f\left(d r^{2}+r^{2} d \Omega_{3}^{2}\right)\right], \\
e^{2 \phi} & =f h^{(n-4) / 2}, \quad C_{1 \cdots n}^{(n)}=\frac{1}{g_{s}} \frac{\left(y^{+}\right)^{2}}{\beta} h f^{-1}, \quad f=1+\frac{N l_{s}^{2}}{r^{2}}
\end{aligned}
$$

which is the type II NS5-brane in the presence of RR $n$-form for $n=0,1,2,3$.

Having a supergravity solution one could proceed to consider the decoupling limit of the theory. The decoupling limit of the NS5-brane can be obtained from D5-brane using S-duality in which $l_{s}^{2} \rightarrow g_{s} l_{s}^{2}$ and $g_{s} \rightarrow g_{s}^{-1}$. Thus the decoupling limit is given by a limit in which $g_{s} \rightarrow 0$ while keeping the following quantities fixed

$$
u=\frac{r}{g_{s} l_{s}^{2}}, \quad b=\frac{\beta}{g_{s} l_{s}^{2}}, \quad l_{s}=\text { fixed }
$$

which is very similar to the decoupling limit of the little string theory [49] (see also [50]). In this limit the supergravity solutions (28) and (29) read

$$
\begin{aligned}
d s^{2}= & h^{-1 / 2}\left[-2 d y^{+} d y^{-}+h\left(\left(y^{+}\right)^{2} d y^{2}+\sum_{i=1}^{n} d x_{i}^{2}\right)+\sum_{j=n+1}^{3} d x_{j}^{2}\right. \\
& \left.+\frac{N l_{s}^{2}}{u^{2}}\left(d u^{2}+u^{2} d \Omega_{3}^{2}\right)\right], \\
e^{2 \phi}= & \frac{N l_{s}^{2}}{u^{2}} h^{(n-3) / 2}, \quad C_{y 1 \cdots n}^{(n+1)}=\frac{l_{s}^{2}}{b} \frac{\left(y^{+}\right)^{2} u^{2}}{N} h,
\end{aligned}
$$

\footnotetext{
${ }^{4}$ Under S-duality we have $\phi \rightarrow-\phi$ and $d s^{2} \rightarrow e^{-\phi} d s^{2}$ where $d s^{2}$ is the metric in string frame. Moreover NS B field gets change to the RR 2-form.

${ }^{5}$ Note that this is not the case for the noncummutative field theory with constant noncommutativity parameter.

${ }^{6}$ To make $u$ of dimension of energy, we have also added $l_{s}$ in the definition of $u$, similarly for $b$.
} 
for (28), and

$$
\begin{aligned}
d s^{2} & =h^{-1 / 2}\left[-2 d y^{+} d y^{-}+\frac{d y^{2}}{\left(y^{+}\right)^{2}}+h \sum_{i=1}^{n} d x_{i}^{2}+\sum_{j=n+1}^{3} d x_{j}^{2}+\frac{N l_{s}^{2}}{u^{2}}\left(d u^{2}+u^{2} d \Omega_{3}^{2}\right)\right] \\
e^{2 \phi} & =\frac{N l_{s}^{2}}{u^{2}} h^{(n-4) / 2}, \quad C_{1 \cdots n}^{(n)}=\frac{l_{s}^{2}}{b} \frac{\left(y^{+}\right)^{2} u^{2}}{N} h
\end{aligned}
$$

for $(29)$. Here $h^{-1}=1+\left(y^{+}\right)^{2} u^{2} / N b^{2}$.

The curvature of the above supergravity solutions reads

$$
l_{s}^{2} \mathcal{R} \sim \frac{1}{\left(1+\frac{\left(y^{+}\right)^{2} u^{2}}{b^{2} N}\right)^{1 / 2}}
$$

therefore for large $u$ the curvature is small and the supergravity solution provides a good description of the theory.

The same as previous section one can consider scalar field scattering of the NS5brane. The corresponding wave equation for an ansatz of the form $\Psi\left(r, y^{+}, y^{-}\right)=$ $\frac{\varphi(r)}{\sqrt{y^{+}}} e^{\frac{i \omega}{\sqrt{2}}\left(y^{+}+Y^{-}\right)}$leads to the following Schrödinger-like equation

$$
\partial_{\rho}^{2} \varphi(\rho)+V_{p}(\rho) \varphi(\rho)=0
$$

where

$$
V_{p}(\rho)=-1+\left(\frac{3}{4}-1\right) \frac{N \omega^{2} l_{s}^{2}}{\rho^{2}},
$$

with $\rho=\omega r$. Following 51$]$ we conclude that the theory has a mass gap of order

$m_{\text {gap }} \sim 1 / \sqrt{N l_{s}^{2}}$. This is exactly the same mass gap as the one in the little string theory, namely the presence of RR field has not changed the mass gap. Note that in the ODp-theory the mass gap will change because of the presence of RR field. In fact in this case the mass gap is given by noncommutative effective parameter which is given by the value of electric RR field [46]. In this sense these theories are closer to the little string theory than ODp-theory.

\section{M-theory 5-brane}

The supergravity solution of M5-brane in the presence of nonzero $C$ 3-form along the worldvolume of the brane has been considered in [41]. This worldvolume theory in the decoupling limit is a well-defined quantum theory with light open membrane, known as OM-theory [30]. M5-brane solution in the presence of $\mathrm{C}$ field with two legs along the worldvolume directions has also been studied in [32] in the context of noncommutative "discpole" theory [52].

In this section we would like to study the M5-brane probing an 11-dimensional time-dependent orbifold background similar to (4). To find the supergravity solution, one can start from D4-brane solution and then lift it up to the 11-dimensional 
supergravity. By making use of the relation between 11-dimensional supergravity solution and its corresponding 10-dimensional dimensional solution

$$
d s_{11}^{2}=e^{\frac{4 \phi}{3}}\left(d x_{11}+C_{\mu} d x^{\mu}\right)^{2}+e^{-\frac{2 \phi}{3}} d s_{10}^{2}
$$

one can lift up the supergravity solution of D4-brane in (11), to find

$$
\begin{aligned}
d s^{2} & =(h f)^{-1 / 3}\left[-2 d y^{+} d y^{-}+h\left(\left(y^{+}\right)^{2} d y^{2}+d x_{1}^{2}+d x_{2}^{2}\right)+d x_{3}^{2}+f\left(d r^{2}+r^{2} d \Omega_{4}^{2}\right)\right], \\
C_{y 12} & =\frac{\left(y^{+}\right)^{2}}{\beta} h f^{-1}, \quad f=1+\frac{\pi N l_{p}^{3}}{r^{3}}, \quad h^{-1}=1+\frac{\left(y^{+}\right)^{2}}{\beta^{2}} f^{-1} .
\end{aligned}
$$

where $l_{p}$ is 11-dimensional Plank length. Of course we have another $C$ form representing the charge of $N$ M5-branes. We note also that under reduction to 10dimensional solution along a transverse direction we will get the type IIA NS5-brane solution in the presence of RR 3-form as in (28).

The decoupling limit of the theory is defined by a limit in which $l_{p} \rightarrow 0$ while keeping the following quantities fixed

$$
u^{2}=\frac{r}{l_{p}^{3}}, \quad b^{2}=\frac{\beta}{l_{p}^{3}} .
$$

In this limit the supergravity solution (37) reads

$$
\begin{gathered}
l_{p}^{-2} d s^{2}=\frac{u^{2}}{(\pi N)^{1 / 3}} h^{-1 / 3}\left[-2 d y^{+} d y^{-}+h\left(\left(y^{+}\right)^{2} d y^{2}+d x_{1}^{2}+d x_{2}^{2}\right)+d x_{3}^{2}\right. \\
\left.+\frac{\pi N}{u^{4}}\left(4 d u^{2}+u^{2} d \Omega_{4}^{2}\right)\right], \\
C_{y 12=} \frac{l_{p}^{3}}{b} \frac{\left(y^{+}\right)^{2} u^{6}}{\pi N} h, \quad h^{-1}=1+\frac{\left(y^{+}\right)^{2} u^{6}}{\pi N b^{4}} .
\end{gathered}
$$

The curvature of the above metric reads

$$
l_{p}^{2} \mathcal{R} \sim \frac{1}{N^{2 / 3}} \frac{1}{\left(1+\frac{\left(y^{+}\right)^{2} u^{6}}{\pi N b^{4}}\right)^{1 / 3}} .
$$

Therefore we can trust the supergravity solution in the UV for a given time. On the other hand under time evolution the curvature decreases and thus the good description is given in terms of supergravity.

\section{Conclusions}

In this paper we have studied supergravity solution of various type II superstring branes in a time-dependent background. The background we have probed by branes is null-brane geometry which is obtained by space-time orbifold accompanied by a 
shift. The shift will resolve the singularities which could appear because of closed time-like loops.

We have seen that the worldvolume theory decouple from gravity for Dp-brane with $p \leq 5$. Following 28 these backgrounds could provide gravity description of noncommutative field theories with time-dependent noncommutativity parameter. These backgrounds preserve 8 supersymmetries out of 32 supersymmetries presented in the flat space. Indeed the time-dependent orbifold we have considered in this paper breaks one-half of the supersymmetry. Adding brane in the theory will break one-half more. Therefore the noncommutative field theories we have studied in this paper preserve only one half of the supersymmetry of the corresponding noncommutative field theories with constant noncommutativity parameter. In this sense the situation is very similar to the noncommutative dipole field theory where the maximal possible SUSY is 8 supercharges [31, 53, 32].

We have also considered type II NS5-branes in the null-brane background. In the decoupling limit we have found a series of six dimensional theories which are identified by an RR form. These theories can be thought as a new deformation of the little string theory. The structure of these theories reminisces the structure of ODp-theories [30], though, as we have seen, the mass gap of theory is given by string length while in ODp theory it is fixed by deformation parameter or the value of electric RR field at infinity.

We note also that using T-duality we have been able to find a new set of NS5brane solutions in the presence of RR field (29). This could also give another deformation of the little string theory. Now starting from gravity solution (29) with $n=2$ one can use S-duality to find a new gravity solution of D5-brane in a time-dependent background which is different from one in (11). Now using T-duality we will get new Dp-brane solution in the time dependent background. It would be interesting to study this background as well.

Having a gravity description of a theory it is natural to compute the Wilson loop of corresponding field theory using its gravity description [54, 55]. It would therefore be interesting to compute the Wilson loop in the noncommutative gauge theory studied in this paper using its gravity dual (21).

\section{Acknowledgments}

We would like to thank M. M. Sheikh-Jabbari for comments and discussions.

\section{References}

[1] G.T. Horowitz and A.R. Steif, "Singular String Solutions With Nonsingular Initial Data," Phys.Lett. B258 (1991) 91.

[2] J. Figueroa-O'Farrill, "Breaking the M-waves," Class.Quant.Grav. 17 (2000) 2925, hep-th/9904124. 
[3] J. Figueroa-O'Farrill and J. Simon, "Generalized Supersymmetric Fluxbranes," JHEP 0112 (2001) 011, hep-th/0110170.

J. Simon, "The Geometry of Null Rotation Identifications," hep-th/0203112.

[4] J. Khoury, B. A. Ovrut, N. Seiberg, P. J. Steinhardt and N. Turok, "From Big Crunch to Big Bang," Phys.Rev. D65 (2002) 086007, hep-th/0108187.

N. Seiberg, "From Big Crunch To Big Bang - Is It Possible?," hep-th/0201039.

[5] V. Balasubramanian, S. F. Hassan, E. Keski-Vakkuri and A. Naqvi, " A SpaceTime Orbifold: A Toy Model for a Cosmological Singularity," hep-th/0202187.

[6] M. Gutperle and A. Strominger, "Spacelike Branes," JHEP 0204 (2002) 018, hep-th/0202210.

[7] L. Cornalba and M. S. Costa, "A New Cosmological Scenario in String Theory," hep-th/0203031.

[8] N. A. Nekrasov, "Milne Universe, Tachyons, and Quantum Group," hepth/0203112.

[9] A. Sen, "Rolling Tachyon," JHEP 0204 (2002) 048, hep-th/0203211.

[10] E. Kiritsis and B. Pioline, "Strings in homogeneous gravitational waves and null holography," hep-th/0204004.

[11] A. J. Tolley and N. Turok, "Quantum Fields in a Big Crunch/Big Bang Spacetime,"hep-th/0204091.

[12] M. Kruczenski, R.C. Myers, A.W. Peet, "Supergravity S-Branes," JHEP 0205 (2002) 039, hep-th/0204144.

[13] O. Aharony, M. Fabinger, G. Horowitz and E. Silverstein, "Clean TimeDependent String Backgrounds from Bubble Baths," JHEP 0207 (2002) 007, hep-th/0204158.

[14] H. Liu, G. Moore and N. Seiberg, "Strings in a Time-Dependent Orbifold," hep-th/0204168.

[15] S. Elitzur, A. Giveon, D. Kutasov and E. Rabinovici, "From Big Bang to Big Crunch and Beyond," JHEP 0206 (2002) 017, hep-th/0204189.

[16] L. Cornalba, M.S. Costa and C. Kounnas, "A Resolution of the Cosmological Singularity with Orientifolds," Nucl.Phys. B637 (2002) 378, hep-th/0204261.

[17] B. Craps, D. Kutasov and G. Rajesh, "String Propagation in the Presence of Cosmological Singularities," JHEP 0206 (2002) 053, hep-th/0205101. 
[18] V. Balasubramanian and S.F. Ross, "The dual of nothing," hep-th/0205290.

[19] S. Kachru and L. McAllister, "Bouncing Brane Cosmologies from Warped String Compactifications," hep-th/0205209.

[20] D. Birmingham and M. Rinaldi, "Bubbles in Anti-de Sitter Space," hepth/0205246.

[21] A. Lawrence, "On the instability of 3d null singularities," hep-th/0205288.

[22] H. Liu, G. Moore and N. Seiberg, "Strings in Time-Dependent Orbifolds," hepth/0206182.

[23] M. Fabinger and J. McGreevy, " On Smooth Time-Dependent Orbifolds and Null Singularities," hep-th/0206196.

[24] G.T. Horowitz and J. Polchinski, "Instability of Spacelike and Null Orbifold Singularities," hep-th/0206228.

[25] A.M. Ghezelbash and R.B. Mann. "Nutty Bubbles," hep-th/0207123.

[26] A. Buchel, P. Langfelder and J. Walcher, "On Time-dependent Backgrounds in Supergravity and String Theory," hep-th/0207214.

[27] S. Hemming, E. Keski-Vakkuri and P. Kraus, "Strings in the Extended BTZ Spacetime," hep-th/0208003.

[28] A. Hashimoto and S. Sethi, "Holography and String Dynamics in TimeDependent Background," hep-th/0208126.

[29] J. Simon, "Null orbifolds in AdS, Time Dependence and Holography," hepth/0208165.

[30] R. Gopakumar, S. Minwalla, N. Seiberg and A. Strominger, " OM Theory in Diverse Dimensions," JHEP 0008 (2000) 008, hep-th/0006062.

[31] A. Bergman, K. Dasgupta, O. J. Ganor, J. L. Karczmarek and G. Rajesh, "Nonlocal Field Theories and their Gravity duals," Phys. Rev. D65 (2002) 066005, hep-th/0103090.

[32] M. Alishahiha and H. Yavartanoo, "Supergravity Description of the Large N Noncommutative Dipole Field Theories," JHEP 0204 (2002) 031, hepth/0202131.

[33] A. Giveon, M. Porrati and E. Rabinovici, "Target Space Duality in String Theory," Phys.Rept. 244 (1994) 77, hep-th/9401139.

E. Bergshoeff, C.M. Hull and T. Ortin, "Duality in the Type-II Superstring Effective Action," Nucl.Phys. B451 (1995) 547, hep-th/9504081. 
[34] J. Figueroa-O'Farrill and Joan Simon, "Supersymmetric Kaluza-Klein reductions of M2 and M5-branes," hep-th/0208107.

[35] J. Figueroa-O'Farrill and Joan Simon, " Supersymmetric Kaluza-Klein reductions of M-waves and MKK-monopoles," hep-th/0208108.

[36] S.S. Gubser, I.R. Klebanov and A.A. Tseytlin, "String Theory and Classical Absorption by Threebranes", Nucl.Phys. B499 (1997) 217; hep-th/9703040.

[37] S. S. Gubser, I. R. Klebanov, "Absorption by Branes and Schwinger Terms in the World Volume Theory", Phys.Lett. B413 (1997) 41; hep-th/9708005.

[38] M. Alishahiha, H. Ita and Y. Oz, "Graviton Scattering on D6 Branes with B Fields", JHEP 0006 (2000) 002; hep-th/0004011.

[39] J. Maldacena, "The Large N Limit of Superconformal Field Theories and Supergravity ," Adv.Theor.Math.Phys. 2 (1998) 231, hep-th/9711200.

[40] N. Itzhaki, J. M. Maldacena, J. Sonnenschein, S. Yankielowicz, "Supergravity and The Large N Limit of Theories With Sixteen Supercharges", Phys.Rev. D58 (1998) 046004; hep-th/9802042.

[41] M. Alishahiha, Y. Oz and M.M. Sheikh-Jabbari, "Supergravity and Large N Noncommutative Field Theories," JHEP

be 9911 (1999) 007, hep-th/9909215.

[42] T. Harmark and N.A. Obers, "Phase Structure of Non-Commutative Field Theories and Spinning Brane Bound States," JHEP 0003 (2000) 024, hepth/9911169.

J.L.F. Barbon and E. Rabinovici, "On 1/N Corrections to the Entropy of Noncommutative Yang-Mills Theories," JHEP 9912 (1999) 017, hep-th/9910019.

J. X. Lu and S. Roy, "( $\mathrm{p}+1)$-Dimensional Noncommutative Yang-Mills and D(p - 2) Branes," Nucl.Phys. B579 (2000) 229m hep-th/9912165.

R.-G. Cai and N. Ohta, "Noncommutative and Ordinary Super Yang-Mills on (D $(p-2), \mathrm{D} p)$ Bound States," JHEP 0003 (2000) 009, hep-th/0001213.

D. S. Berman, V. L. Campos, M. Cederwall, U. Gran, H. Larsson, M. Nielsen, B. E.W. Nilsson and P. Sundell, "Holographic Noncommutativity," JHEP 0105 (2001) 002, hep-th/0011282.

[43] R. Gopakumar, J. Maldacena, S. Minwalla and A. Strominger, "S-Duality and Noncommutative Gauge Theory," JHEP 0006 (2000) 036, hep-th/0005048.

[44] T. Harmark, "Open Branes in Space-Time Non-Commutative Little String Theory," Nucl.Phys. B593 (2001) 76, hep-th/0007147. 
[45] T. Harmark, "Supergravity and Space-Time Non-Commutative Open String Theory," JHEP 0007 (2000) 043, hep-th/0006023.

[46] M. Alishahiha, "On Type II NS5-branes in the presence of an RR field," Phys.Lett. B486(2000) 194, hep-th/0002198

[47] M. Alishahiha, Y. Oz and J. G. Russo, "Supergravity and Light-Like Noncommutativity," JHEP 0009 (2000) 002, hep-th/0007215.

[48] I. Mitra and S. Roy, "(NS5,Dp) and (NS5,D(p+2),Dp) bound states of type IIB and type IIA string theories," JHEP 0102 (2001) 026, hep-th/0011236

[49] N. Seiberg, "Matrix Description of M-theory on $T^{5}$ and $T^{5} / Z_{2}$," Phys.Lett. B408 (1997) 98, hep-th/9705221.

[50] M. Berkooz, M. Rozali and N. Seiberg, " Matrix Description of M-theory on $T^{4}$ and $T^{5}, "$ Phys.Lett. B408 (1997) 105, hep-th/9704089.

R. Dijkgraaf, E. Verlinde and H. Verlinde, "BPS Quantization of the FiveBrane," Nucl.Phys. B486 (1997) 89, hep-th/9604055.

[51] S. Minwalla and N. Seiberg, "Comments on the IIA NS5-brane," JHEP 9906 (1999) 007, hep-th/9904142.

[52] K. Dasgupta, O. J. Ganor and G. Rajesh, "Vector Deformations of N=4 SuperYang-Mills Theory, Pinned Branes, and Arched Strings," JHEP 0104 (2001) 034, hep-th/0010072.

[53] K. Dasgupta and M. M. Sheikh-Jabbari, "Noncommutative Dipole Field Theories," JHEP 0202 (2002) 002, hep-th/0112064.

[54] J. M. Maldacena, "Wilson loops in Large $N$ Field theories," Phys. Rev. Lett. 80 (1998) 4859, hep-th/9803002.

[55] S.-J. Rey and J. Yee. "Macroscopic String as Heavy Quarks of Large $N$ Gauge theory and Anti-de-Sitter Supergravity," Eur. Phys. J. C22 (2001) 379, hepth/9803001. 